pH-Metrie, fehlende klare Indikationen für die endoskopische Diagnostik, fehlender Nachweis des Nutzens radiologischer Methoden sowie große Interpretationsspielräume bei der Impedanzmessung lassen den Kliniker beinahe orientierungslos erscheinen. Dieser wird sich vorerst an den nicht gut evidenzbasierten Empfehlungen der Fachgesellschaften orientieren [Vandenplas Y et al. J Pediatr Gastroenterol Nutr 2009; 49: 498-547] und bei Kindern mit Warnsymptomen vor allem zur Endoskopie raten, auch um die häufiger werdende eosinophile Ösophagitis zu erkennen. Eine probatorische Therapie mit Protonenpumpenhemmern - wie bei Erwachsenen häufig durchgeführt - ist bei Kindern unzureichend belegt. Die Domäne der $\mathrm{pH}$ Metrie werden zunächst weiterhin die respiratorischen Komplikationen sein. Aber wer weiß, ob das alles richtig ist, was wir tun?

Ob die Forderung der Autoren nach neuen, randomisierten, qualitativ guten diagnostischen Studien sich bald erfüllen wird, bleibt zweifelhaft. Angesichts der auch therapeutisch dürftigen Datenlage [van der Pol RJ et al. Pediatrics 2011; 127: 925-35] kann man hoffen, dass diese Studien dann auch diätetische, medikamentöse und chirurgische Therapieoptionen prüfen.

Fazit: Für die gängigen diagnostischen Instrumente zur Untersuchung des GÖR bei Kindern fehlen Daten zur Indikationsstellung und zur diagnostischen Treffsicherheit, weil die methodische Qualität aller bisherigen Studien dürftig ist.

Dr. Martin Claßen

\section{Der Werdegang von ehemaligen ADHS-Patienten}

\author{
Jungen mit der Diagnose "ADHS" schneiden schlechter in Schule und Beruf \\ ab und haben später einen niedrigeren sozialen und ökonomischen Status als \\ ihre gesunden Altersgenossen. Eine Gruppe von Autoren vom Child Study \\ Center in New York konnte dies anhand der Biografie von ehemaligen ADHS- \\ Patienten belegen.
}

$\mathrm{D}$ ie Autoren hatten in ihre prospektive Verlaufsstudie insgesamt 207 Jungen mit ADHS, aber ohne Störung des Sozialverhaltens im Alter von acht Jahren aufgenommen. Sie verglichen sie mit einer gleichaltrigen Kontrollgruppe ohne ADHS. Bei der letzten Verlaufsuntersuchung - 33 Jahre nach der Erstuntersuchung - nahmen noch $65,2 \%$ teil, im Vergleich zu 76,5\% aus der Kontrollgruppe. Insgesamt wurde der Werdegang von 135 weißen Männern mit ADHS in der Kindheit und 136 weißen Männern ohne ADHS miteinander verglichen. Das mittlere Alter betrug 41 Jahren. Die Autoren untersuchten in der aktuellen Studie nicht nur das pyschosoziale und berufliche Outcome, sondern differenzierten auch hinsichtlich noch bestehender ADHS-Symptomatik.

Im Vergleich zur Kontrollgruppe hatten die Probanden, die als Kind mit ADHS diagnostiziert worden waren, eine signifikant schlechtere Abschlussnote beim Schulabschluss bei um zwei Jahre kürzerer Schuldauer. 12,6\% der ehemaligen ADHS-Patienten erreichten nicht den Highschool-Abschluss versus $0,7 \%$ aus der Kontrollgruppe. Den Bachelor-Abschluss machten nur 15,6\% versus 34,6\%, einen höheren Studienabschluss erlangten nur $3,7 \%$ versus $29,4 \%$.
Dies wirkte sich auf das Einkommen aus: Das durchschnittliche Jahresgehalt lag in der ADHS-Gruppe mit 60.000 USDollar deutlich unter dem der Kontrollgruppe mit 100.000 US-Dollar. Erfreulicherweise fanden sich $83,7 \%$ der Probanden in einem Arbeitsverhältnis, in der Placebo-Gruppe waren es 94,9\%.

Auf Beeinträchtigungen im sozialen Status wies die hohe Scheidungsrate unter den ehemaligen ADHS-Kindern von $31,1 \%$ im Gegensatz zu 11,8\% in der Kontrollgruppe hin. 3,7\% der Probanden hatten eine Haftstrafe hinter sich, $7,2 \%$ waren bis zum Alter von 42 Jahren bereits verstorben. In der Kontrollgruppe lagen diese Werte bei $0,7 \%$ bzw. $2,8 \%$.

Während sich Alkoholabusus in den Gruppen nicht signifikant unterschied, beobachten die Autoren einen signifikant höhere Substanz-/Nikotinabhängigkeit von $14,1 \% / 30,4 \%$ versus $5,1 \% /$ $8,8 \%$. Außerdem bestand eine Tendenz zu mehr Angststörungen und Depressionen bei den ehemaligen ADHS-Patienten. Auch trat eine antisoziale Persönlichkeitsstörung mit $16,2 \%$ versus $0 \%$ deutlich höher auf.

Die Autoren stellten sich dann die Frage, ob die Ergebnisse abhängig vom Persistieren der ADHS noch im Erwachsenenalter sind: Lediglich im Bereich der antisozialen Persönlichkeitsstörung und der Substanzabhängigkeit zeigten die Probanden mit noch persistierendem ADHS im Erwachsenenalter signifikant höhere Werte. Im Vergleich zur Kontrollgruppe waren diese aber auch bei den Probanden ohne aktuelle ADHS erhöht.

Klein RG et al. Clinical and functional outcome of childhood attention-deficit/hyperactivity disorder 33 years later. Arch Gen Psychiatry 2012; 69: 1295-303

Kommentar: Die Ergebnisse bestätigen die klinische Erfahrung und auch die Befunde anderer Langzeitstudien [Barkley RA et al. The Gilford Press, N.Y 2010]. Erfreulich an den vorliegenden Ergebnissen ist, dass nur ein kleiner Teil (12,6\%) keinen zufriedenstellenden Schulabschluss erreicht und immerhin $83,7 \%$ ein Beschäftigungsverhältnis gefunden haben. Ob diese Werte allerdings eins zu eins auf deutsche Verhältnisse übertragen werden können, ist fraglich. Auch ist dabei zu bedenken, dass die „Hochrisikogruppe", nämlich die Kinder mit ADHS plus Störungen des Sozialverhaltens - die gemäß der MTA-Studie ca. 15\% betragen ausgeschlossen wurde.

Die Autoren folgern aus ihren Ergebnissen, wie wichtig die rechtzeitige Diagnostik und Behandlung der ADHS schon im Kindesalter für das weitere Leben der Betroffenen ist. In vielen Bereichen (z. B. Arbeitsverhältnis, Gehaltsstruktur, antisoziale Persönlichkeitsstörung ohne Substanzmissbrauch und Nikotinabhängigkeit) spielte es im Erwachsenenalter keine Rolle mehr, ob die ADHS noch persistierte, hier werden wohl bereits im Kindesalter die entscheidenden Weichen gestellt. Dr. Kirsten Stollhoff 